\title{
Resveratrol attenuates inflammation in the rat heart subjected to ischemia-reperfusion: Role of the TLR4/NF- $\mathrm{KB}$ signaling pathway
}

\author{
JINGBO LI* , CHUNYANG XIE* , JUNLI ZHUANG, HALI LI, YE YAO, CHANGGANG SHAO and HAIYANG WANG \\ Division of Vascular Surgery, Department of General Surgery, The First Affiliated Hospital of Harbin Medical University, \\ Harbin, Heilongjiang 150001, P.R. China
}

Received December 12, 2013; Accepted June 24, 2014

DOI: $10.3892 / \mathrm{mmr} .2014 .2955$

\begin{abstract}
It has been previously reported that Toll-like receptor 4 (TLR4)/NF- $\kappa \mathrm{B}$ signaling mediates early inflammation during myocardial ischemia and reperfusion. It has additionally been suggested that resveratrol produces cardioprotective and anti-inflammatory effects. The aim of the present study was to investigate whether resveratrol could modulate TLR4/NF- $\kappa \mathrm{B}$ signaling, reduce neutrophil accumulation and TNF- $\alpha$ induction in an ischemia/reperfusion injured rat heart model. Rats were randomly exposed to a sham operation, myocardial ischemia and reperfusion $(\mathrm{MI} / \mathrm{R}), \mathrm{MI} / \mathrm{R}+$ resveratrol or $\mathrm{MI} / \mathrm{R}+$ resveratrol + L-NAME. The data showed that following MI/R, the expression of myocardial TLR4 and NF- $\mathrm{NB}$ increased significantly in the area of induced ischemia. As compared with MI/R, resveratrol significantly attenuated the expression of TLR4 and $\mathrm{NF}-\kappa \mathrm{B}$ and reduced the levels of myeloperoxidase, serum and myocardial TNF- $\alpha$ production, myocardial infarct size and myocardial apoptosis induced by MI/R. All the effects of resveratrol were abolished upon application of L-NAME, a nitric oxide (NO) synthase inhibitor. These data provide evidence that resveratrol inhibits TLR4/NF- $\mathrm{B}$ signaling in the rat heart subjected to $\mathrm{MI} / \mathrm{R}$, and the anti-inflammatory effect of resveratrol is associated with $\mathrm{NO}$ production.
\end{abstract}

\section{Introduction}

The inflammatory reaction induced by ischemia/reperfusion is one of the most significant effects to occur in myocardial ischemia-reperfusion injury (1). During the process of inflammation, various cytokines are released, including tumor necrosis factor- $\alpha$ (TNF- $\alpha$ ), interleukin-6 (IL-6) and IL-8 (2). TNF- $\alpha$ can trigger the inflammatory reaction caused by myocardial

Correspondence to: Dr Haiyang Wang, Division of Vascular Surgery, Department of General Surgery, The First Affiliated Hospital of Harbin Medical University, 23 Youzheng Street, Harbin, Heilongjiang 150001, P.R. China

E-mail: wanghaiyang2014@163.com

*Contributed equally

Key words: resveratrol, ischemia/reperfusion injury, TLR4/NF-кB signaling, neutrophil, TNF- $\alpha$ ischemia-reperfusion (MI/R). Vascular endothelial cell injury, and inflammatory cells, such as neutrophils activated by cytokines, and adhesion molecules are also involved in the inflammatory response. TNF- $\alpha$ activity and the amount of neutrophil infiltration can be considered as indicators of the inflammatory reaction. It has been previously demonstrated that Toll-like receptor 4 (TLR4)/NF- $\kappa \mathrm{B}$ signaling is activated during $\mathrm{MI} / \mathrm{R}$, which in turn results in TNF- $\alpha$ induction, thus initiating an inflammatory reaction during $\mathrm{MI} / \mathrm{R}(3,4)$.

The TLRs are a family of molecules that function in the process of innate immunity $(5,6)$. Activation of the corresponding TLRs triggers an inflammatory response, alerting the host to the presence of microbial invasion and initiating an immune response. Previous studies have demonstrated that some TLR family members can signal the presence of tissue damage to the host due to activation by endogenous molecules released from damaged tissues $(7,8)$. Heparan sulfate, hyaluronic acid and other endogenous molecules have been shown to initiate inflammatory pathways through TLR4 (9-11). It has been suggested that TLR4-mediated signaling induces myocardial dysfunction during MI/R (12).

Resveratrol (Res) is a polyphenolic compound, which is predominantly naturally occurring in red grapes and wine. In a previous epidemiological study (13) investigating the association between eating habits and coronary heart disease, a phenomenon was proposed that in all developed countries, the French consume the most quantity of wine on average, but have the lowest morbidity of coronary heart disease. This phenomenon is termed the 'French paradox', and has been attributed to the benefits acquired from Res present in wine. Res has extensive pharmacological effects, including anticancer properties (14-17), improving ifosfamide-induced Fanconi syndrome in rats (18), treating diabetic nephropathy (19) and neuronal protection $(20,21)$. Though previous studies have suggested that Res has an anti-inflammatory effect, the role of Res in mediating inflammation remains to be understood (22). The present study aims to investigate the role of the TLR4/NF- $\mathrm{B}$ signaling pathway in the anti-inflammatory effect of Res in a rat model of MI/R.

\section{Materials and methods}

Reagents. Res and L-NAME were purchased from Sigma-Aldrich (St. Louis, MO, USA). The myeloperoxidase (MPO) assay 
kit was purchased from Jiancheng Bioengineering Institute (Nanjing, China). The TNF- $\alpha$ ELISA kit was purchased from R\&D Systems (Minneapolis, MN, USA). The bicinchoninic acid (BCA) protein quantification kit was purchased from Bio-Rad (Hercules, CA, USA). NF-кB p65 mouse monoclonal antibody (L8F6) was purchased from Cell Signaling Technology, Inc. (Danvers, MA, USA). The anti-TLR4 antibody (ab13556) and goat anti-rabbit immunoglobulin ( $\mathrm{IgG})$ were purchased from Abcam (Cambridge, MA, USA). EnVison ${ }^{\mathrm{TM}}$ was purchased from Gene Tech (Shanghai, China) and the cell death detection kit was purchased from Roche Diagnostics (Mannheim, Germany).

Animals. Forty adult male Sprague Dawley rats (250-300 g) were purchased from the Center of Experimental Animals in the Harbin Medical University (Harbin, Heilongjiang, China). All animals used in this study were cared for in accordance with the Guide for the Care and Use of Laboratory Animals published by the United States National Institute of Health (NIH publication no. 85-23, revised 1996). All procedures were approved by the Committee of Experimental Animals of Harbin Medical University.

MI/R model and experimental protocol. Male Sprague Dawley rats (250-300 g) were anesthetized intraperitoneally with sodium pentobarbital (Sigma-Aldrich; $40 \mathrm{mg} / \mathrm{kg}$ ). Myocardial ischemia was produced by exteriorizing the heart with a left thoracic incision followed by a slipknot (5-0 silk) around the left anterior descending coronary artery (LAD). After $30 \mathrm{~min}$ of ischemia, the slipknot was released and the animal received $120 \mathrm{~min}$ of reperfusion.

Rats were randomly assigned to four experimental groups, with 10 rats in each group: i) sham group: silk was drilled underneath the LAD but the LAD was not ligated; ii) $\mathrm{MI} / \mathrm{R}$ group: LAD was ligated for $30 \mathrm{~min}$ followed by 120 min reperfusion together with receiving vehicle $(0.9 \%$ $\mathrm{NaCl}$ i.v.); iii) $\mathrm{MI} / \mathrm{R}+\mathrm{Res}$ group: Res [100 $\mu \mathrm{mol} / \mathrm{l}$, intravenous (i.v)] was administered 5 min prior to reperfusion; iv) $\mathrm{MI} / \mathrm{R}+\mathrm{Res}+\mathrm{L}-\mathrm{NAME}$ group: L-NAME (1 mmol/l, i.v.), a nitric oxide (NO) synthase inhibitor, was administered $20 \mathrm{~min}$ prior to reperfusion. Res $(100 \mu \mathrm{mol} / \mathrm{l}$, i.v) was administered fifteen minutes following L-NAME treatment.

Assay of myocardial infarct area. Following reperfusion, the myocardial infarct size was determined by a double-staining technique and a digital imaging system (infarct area/area at risk $\times 100 \%$ ) (23). Following reperfusion, the coronary blood flow was again blocked and Evans blue (2\%, $4 \mathrm{ml})$ was injected and rapidly distributed by the right ventricle into the body. The heart was then quickly removed and cryopreserved at $-20^{\circ} \mathrm{C}$. The heart was cut into $1 \mathrm{~mm}$ slices, placed in 1\% 2,3,5-triphenyltetrazolium chloride (TTC) solution, incubated for $15 \mathrm{~min}$, and then placed in $4 \%$ formaldehyde solution overnight. The Evans blue (blue staining, non-ischemic area), TTC (red staining, ischemic area) and non-TTC-stained areas (white, infarct area) were analyzed using a computerized digital imaging system. The myocardial infarct area (infarct area/area at risk \%, INF/AAR\%) was subsequently calculated.

Determination of myocardial apoptosis. Following reperfusion, myocardial apoptosis was analyzed by terminal deoxynucleotidyl transferase dUTP nick end labeling assay (TUNEL) using an in situ cell death detection kit. A doublestaining technique was used. TUNEL staining for apoptotic cell nuclei and DAPI staining for all myocardial cell nuclei were performed as described previously (24). The index of apoptosis was expressed as the number of positively stained apoptotic myocytes/the total number of myocytes counted x100\%.

Immunohistochemical analysis of myocardial TLR4 expression. Prior to immunohistochemical examination, $3 \mu \mathrm{m}$ slices from pretreated myocardium tissue were placed in a bathing solution of $3 \% \mathrm{H}_{2} \mathrm{O}_{2}$ and $60 \%$ methanol in phosphate-buffered saline (PBS) for $30 \mathrm{~min}$ and then treated with $0.01 \mathrm{~mol} / 1$ sodium citrate buffer at $95^{\circ} \mathrm{C}$ in a microwave oven for $13 \mathrm{~min}$, for antigen retrieval. The specimens were then treated with $5 \%$ normal goat serum and 5\% bovine serum albumin (BSA) in PBS. Prior to each step, the sections were rinsed three times in PBS. Incubation with primary TLR4 antibody was performed in a PBS-based solution of $1 \% \mathrm{BSA}$ for $12 \mathrm{~h}$ at $4^{\circ} \mathrm{C}$ at the recommended dilutions. After rinsing with $\mathrm{PBS}$, sections were incubated with the corresponding secondary biotinylated goat anti-rabbit EnVison ${ }^{\mathrm{TM}}$ antibodies for $1 \mathrm{~h}$ at room temperature. A streptavidin/horseradish peroxidase complex was then applied as a detection system (1:100 dilution) for $1 \mathrm{~h}$. Finally, staining was developed with 3,3-diaminobenzidine tetra-hydrochloride in $0.05 \mathrm{~mol} / 1$ Tris- $\mathrm{HCl}$ buffer and $0.1 \% \mathrm{H}_{2} \mathrm{O}_{2}$. Negative control sections were incubated without the primary antibody. All the samples in this study were analyzed by Image Pro Plus software (Media Cybernetics, Inc., Rockville, MD, USA).

Western blotting analysis of myocardial $N F-\kappa B$ expression. Proteins were extracted from heart tissue and the immunoblots were probed with anti-NF- $\kappa \mathrm{B}$ antibodies overnight at $4^{\circ} \mathrm{C}$ followed by incubation with the corresponding secondary antibodies at room temperature for $1 \mathrm{~h}$. The blots were visualized with ECL-Plus reagent.

Determination of MPO level. Following reperfusion, the myocardial tissue was placed at $-70^{\circ} \mathrm{C}$ for preservation. The MPO test kit was used to detect the level of MPO in the myocardial tissue according to the manufacturer's instructions.

Detection of TNF- $\alpha$ level. Following reperfusion, the levels of TNF- $\alpha$ in the myocardial tissue homogenate and serum were detected according to the manufacturer's instructions. A BCA kit was used for quantification of the protein samples.

Statistical analysis. The data are presented as the mean \pm SD. The significance of differences among groups was evaluated by a Student's t-test for unpaired data or Dunnett's t-test for multiple comparisons preceded by one-way analysis of variance. $\mathrm{P}<0.05$ was considered to indicate a statistically significant difference.

\section{Results}

Res reduces the area of myocardial infarction induced by $M I / R$. MI/R induced a significant area of infarction. As compared with the MI/R group, Res significantly reduced the 
A

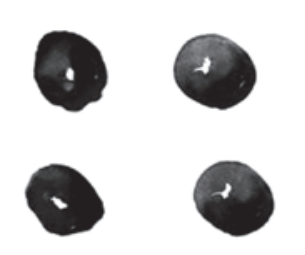

B

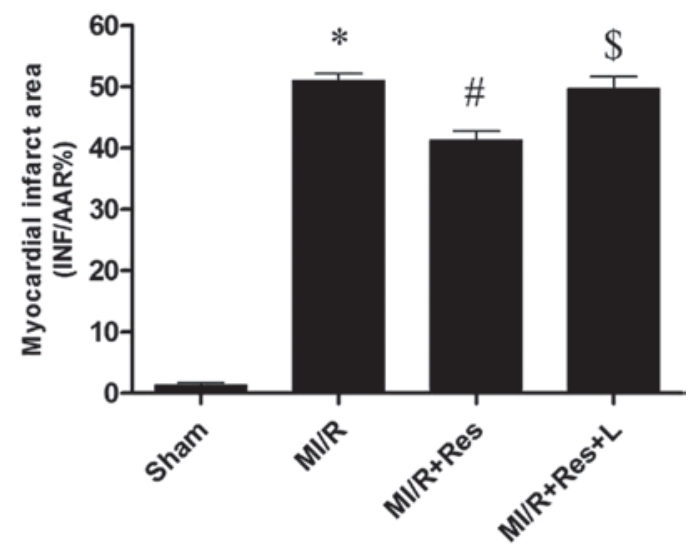

Figure 1. Comparison of myocardial infarct. (A) Representative 2,3,5-triphenyltetrazolium chloride-Evans blue double staining of samples from rat ventricles subjected to different treatments. (B) Quantitative analysis of myocardial infarct sizes (INF/AAR\%). Error bars represent the mean \pm standard deviation. ${ }^{*} \mathrm{P}<0.05$ vs. Sham; ${ }^{\text {P }}<0.05$ vs. MI/R; ${ }^{~} \mathrm{P}<0.05$ vs. MI/R + L. Sham, sham group; MI/R, MI/R group; MI/R + Res, MI/R + resveratrol group; MI/R + Res + L-NAME, $\mathrm{MI} / \mathrm{R}+$ resveratrol + L-NAME group; MI, myocardial ischemia; $\mathrm{R}$, reperfusion; Res, resveratrol.

area of myocardial infarction. The effect of Res was blocked by L-NAME, a nitric oxide synthase (NOS) inhibitor (Fig. 1).

Res reduces myocardial apoptosis in rat hearts subjected to MI/R. As shown in Fig. 2, a low level of TUNEL-positive staining was detected in the sham group whereas a significant number of TUNEL-positive cells were observed in the MI/R group ( $* \mathrm{P}<0.0001)$. Administration of Res exerted a significant anti-apoptotic effect $\left({ }^{\#} \mathrm{P}=0.0022\right)$, which was abolished by L-NAME ( $\left.{ }^{\$} \mathrm{P}=0.0025\right)$.

Res reduces TLR4 protein expression in rat hearts subjected to $M I / R$. Immunohistochemical analysis (Fig. 3) showed that the expression of TLR4 was markedly increased in the the $\mathrm{MI} / \mathrm{R}$ group $(\mathrm{P}<0.00015)$ compared with the sham group. Upon administration of Res, the expression of TLR4 was significantly reduced $(\mathrm{P}=0.0029)$ and this effect was abolished by L-NAME $(\mathrm{P}=0.0035)$.

Res decreases $N F-\kappa B$ expression in rat hearts subjected to $M I / R$. The expression of NF- $\mathrm{KB}$, a downstream molecule of TLR4, was determined in order to further study the effects of Res on TLR4 mediated signaling. Western blot analysis (Fig. 4) indicated that the expression of NF- $\mathrm{kB}$ was upregulated in $\mathrm{MI} / \mathrm{R}$ group (0.0052). Administration of Res significantly decreased NF- $\kappa B$ expression $\left({ }^{\#} \mathrm{P}=0.0082\right)$ and the effect of Res was abolished by L-NAME ( $\left.{ }^{\$} \mathrm{P}=0.0065\right)$.

Res inhibits neutrophil infiltration in MI/R tissue. Neutrophils contain a certain amount of MPO, accounting for 5\% of the dry cell weight. The activity of MPO in the myocardium may therefore be considered as an indication of neutrophil infiltration. As shown in Fig. 5, the MPO activity in the sham group was relatively lower, whereas the MPO activity in the $\mathrm{MI} / \mathrm{R}$ group was significantly increased $(\mathrm{P}<0.0001)$. Res significantly decreased myocardial MPO activity $(\mathrm{P}=0.0042)$, whereas L-NAME attenuated the effects of Res $(\mathrm{P}=0.0175)$.

Res reduces TNF- $\alpha$ levels in the serum and MI/R tissue. $\mathrm{MI} / \mathrm{R}$ injury results in the production of a large amount of TNF- $\alpha$. Myocardial and serum TNF- $\alpha$ levels were therefore examined. As shown in Fig. 6, as compared with the MI/R group, Res significantly decreased the levels of TNF- $\alpha$ in both the myocardium and serum, and these effects were eliminated by L-NAME.

\section{Discussion}

The present study identified the following: i) Res attenuates the inflammatory reaction induced by $I / R$ injury by inhibiting TLR4/NF- $\kappa B$ signaling; ii) Res attenuates the inflammatory reaction induced by $\mathrm{I} / \mathrm{R}$ injury through inhibiting neutrophil infiltration and TNF- $\alpha$ production; iii) NO may function in the protective mechanism of Res.

The inflammatory reaction has an important function in MI/R injury (1). The release of inflammatory cytokines and the aggregation and infiltration of inflammatory cells are considered as the key steps in inflammation (25).

TNF- $\alpha$ is predominantly secreted by macrophages, and is likely to promote an inflammatory cascade by increasing the release of other proinflammatory cytokines and influencing neutrophil recruitment (26). TNF- $\alpha$ is an important cytokine in inflammation and functions in the initiation of the inflammatory response induced by MI/R (27). TNF- $\alpha$ can induce the release of other inflammatory mediators, increase the expression of cell adhesion factors, and promote neutrophil adhesion to endothelial cells. In addition, TNF- $\alpha$ has a negative inotropic effect, which can inhibit myocardial contractility, and lower blood pressure. TNF- $\alpha$ can also induce cardiomyocyte apoptosis and participate in ventricular remodeling (28). Previous studies have suggested that the level of TNF- $\alpha$ significantly increases following MI/R (29), whereas the administration of TNF- $\alpha$ monoclonal antibody was shown to attenuate edema, and is conducive to cardiac function recovery (30).

$\mathrm{MI} / \mathrm{R}$ injury is induced in part by neutrophil activation. The underlying mechanisms include: i) Cell damage caused by the release of oxygen free radicals, proteolytic enzymes, and cytotoxic substances; ii) the released inflammatory mediators cause vascular endothelial cell damage, increased vascular permeability, and edema; iii) additional activation of inflammatory cells increase further the inflammatory response (31); iv) neutrophil adhesion to the vascular endothelium and small 
A
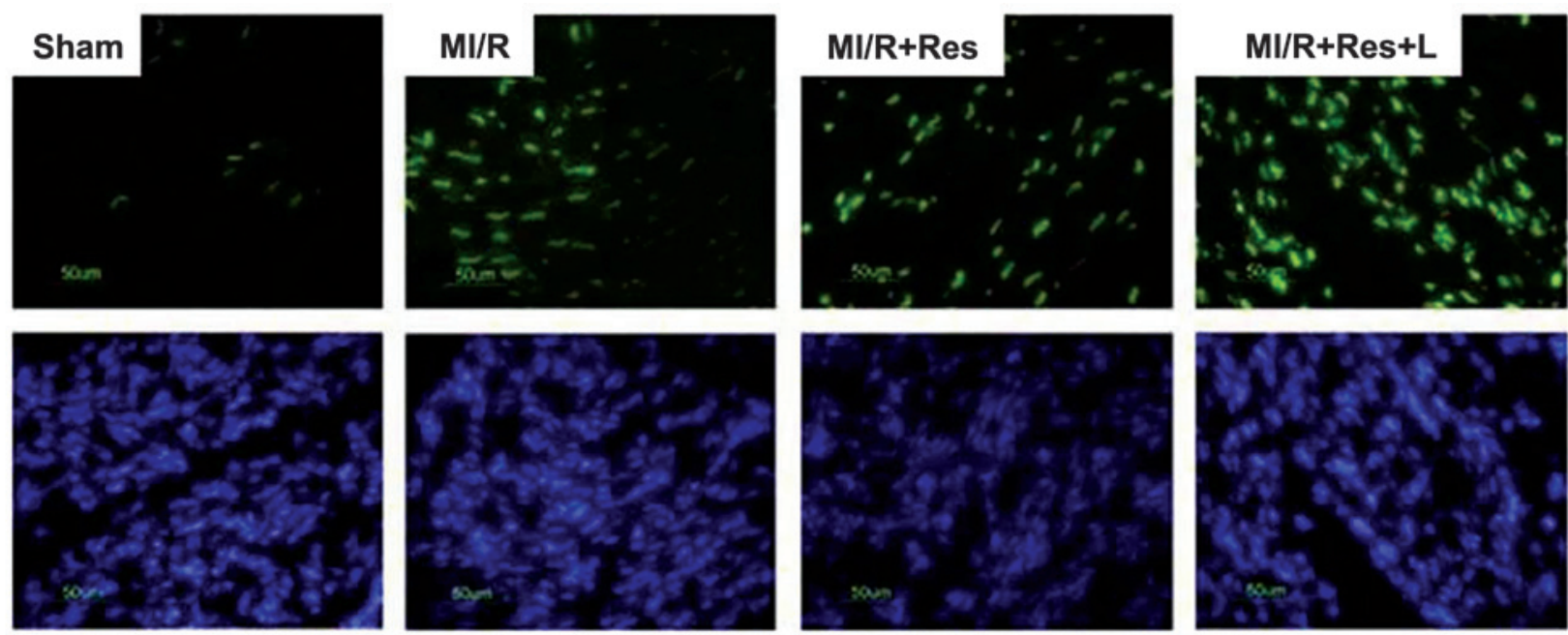

B

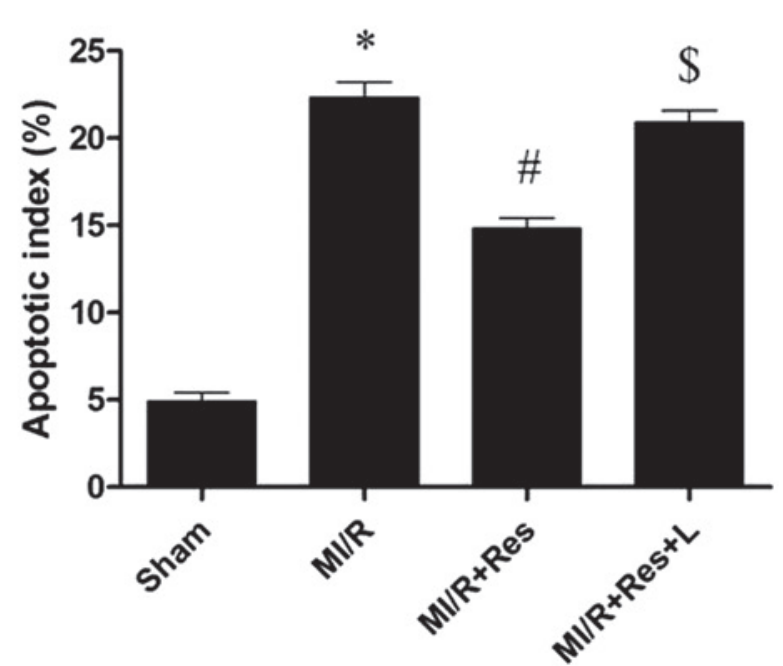

Figure 2. Apoptosis assay. (A) TUNEL staining. (B) Quantitative analysis of percentage of cardiomyocytes undergoing apoptosis. Error bars represent the mean \pm standard deviation. " $\mathrm{P}<0.05$ vs. Sham; ${ }^{*} \mathrm{P}<0.05$ vs. MI/R; ${ }^{~} \mathrm{P}<0.05$ vs. MI/R + L. Sham, sham group; MI/R, MI/R group; MI/R + Res, MI/R + resveratrol group; MI/R + Res + L-NAME, MI/R + resveratrol + L-NAME group; MI, myocardial ischemia; R, reperfusion; Res, resveratrol.
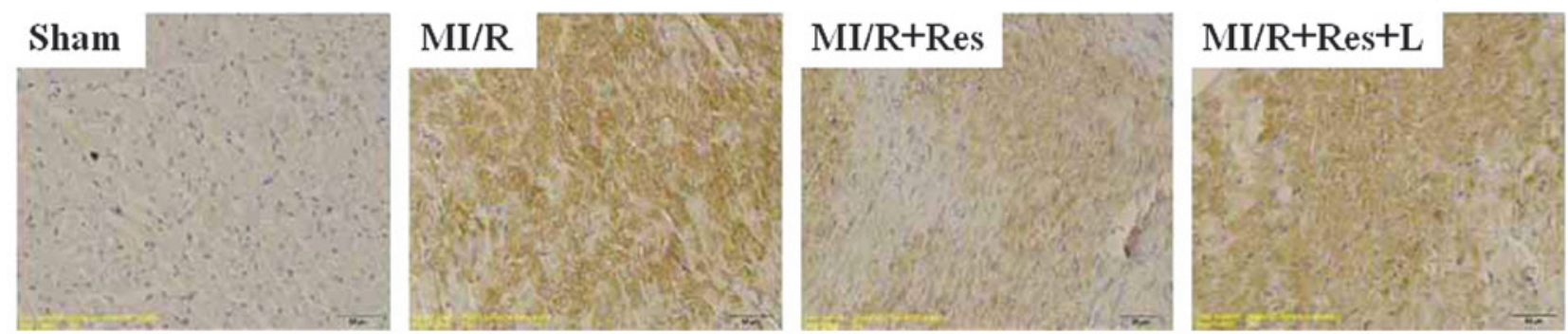

Figure 3. Immunohistochemical analysis of myocardial Toll-like receptor 4 (TLR4) expression. TLR4 is stained brown. Sham, sham group; MI/R, MI/R group; MI/R + Res, MI/R + resveratrol group; MI/R + Res + L-NAME, MI/R + resveratrol + L-NAME group; MI, myocardial ischemia; R, reperfusion; Res, resveratrol.

blood vessel occlusion results in a no-reflow phenomenon. Previous studies have identified the mechanistic link between neutrophil activation and I/R injury. Removal of neutrophils or drug inhibition of neutrophil activity has been shown to reduce ischemia/reperfusion injury $(32,33)$. In the present study, it was identified that neutrophil accumulation and TNF- $\alpha$ production in the MI/R group was significantly increased. It was observed that Res reduced neutrophil accumulation and TNF- $\alpha$ production, therefore indicating that Res inhibited neutrophil accumulation and TNF- $\alpha$ production and attenuated neutrophil-mediated I/R injury.

TLRs are present in both immune and non-immune cells, and their expression is rapidly altered in response to pathogens, cytokines, and environmental stressors (34). The functions of TLRs, including TLR4, are responsible for the genesis of various cardiovascular disorders as well as the activation of $\mathrm{NF}-\kappa \mathrm{B}$ and inflammatory cytokines, including TNF- $\alpha$, which results in cell death $(35,36)$. TLR4 is expressed in numerous 


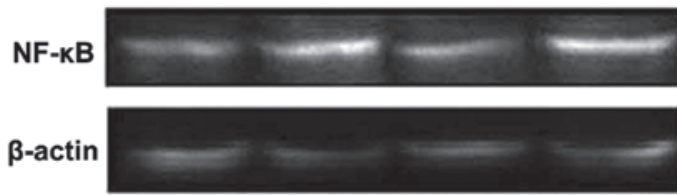

B

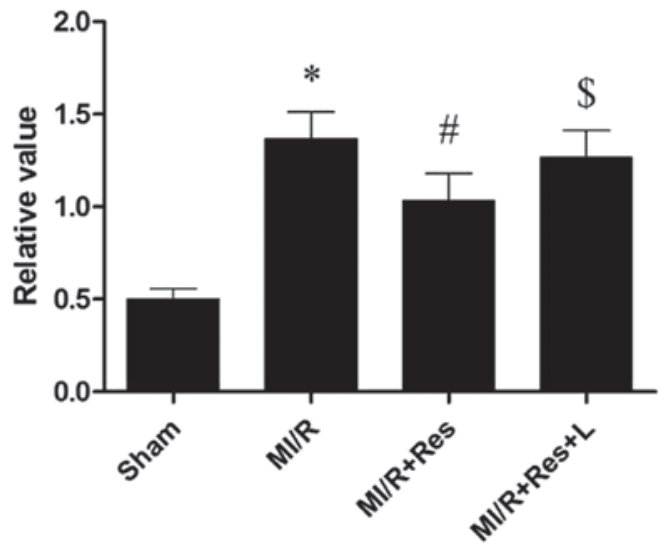

Figure 4. Effect of resveratrol on NF- $\mathrm{BB}$ expression. (A) NF- $\mathrm{kB}$ expression detected by western blotting. (B) Quantitative analysis of NF- $\kappa$ B. Error bars represent the mean \pm standard deviation. ${ }^{*} \mathrm{P}<0.05$ vs. Sham, ${ }^{*} \mathrm{P}<0.05$ vs. MI/R, ${ }^{\$} \mathrm{P}<0.05$ vs. MI/R + L. Sham, sham group; MI/R, MI/R group; $\mathrm{MI} / \mathrm{R}+$ Res, $\mathrm{MI} / \mathrm{R}$ + resveratrol group; MI/R + Res + L-NAME, MI/R + resveratrol + L-NAME group; MI, myocardial ischemia; $R$, reperfusion; Res, resveratrol.

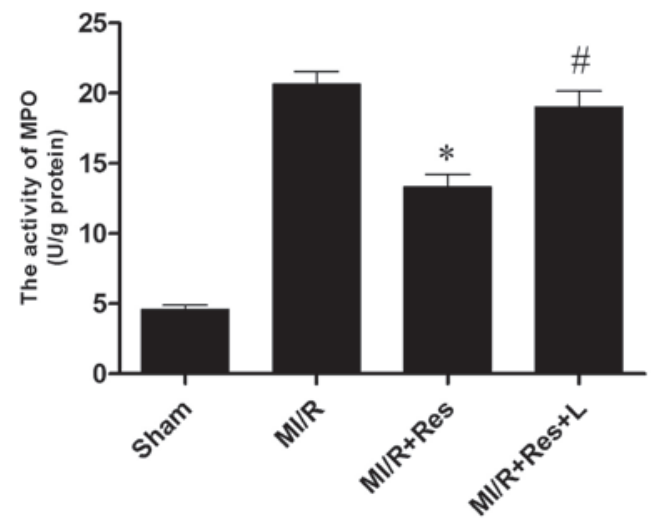

Figure 5. Comparison of MPO activity in each group. As compared with $\mathrm{MI} / \mathrm{R}$ group, the MPO activity in MI/R + Res group reduced significantly. L-NAME eliminated the effect of resveratrol. Error bars represent the mean \pm standard deviation. ${ }^{*} \mathrm{P}<0.05$ vs. Sham, ${ }^{\#} \mathrm{P}<0.05$ vs. MI/R. Sham, sham group; MI/R, MI/R group; MI/R + Res, MI/R + resveratrol group; $\mathrm{MI} / \mathrm{R}+$ Res + L-NAME, MI/R + resveratrol + L-NAME group; MI, myocardial ischemia; $\mathrm{R}$, reperfusion; MPO, myeloperoxidase.

tissues including the heart, where the receptor becomes upregulated in response to ischemia. TLR4 expression levels in cardiac myocytes are enhanced by either lipopolysaccharide or IL-1 (37). The mechanism and function of TLR4-mediated signaling has been well established, and it is considered that the upregulation of TLR4 results in activation of NF- $\kappa \mathrm{B}$ in cardiomyocytes. This can augment the release of cytokines, including TNF- $\alpha$, IL-1 and MCF, as well as the infiltration of leukocytes (38).

It is considered that $\mathrm{NO}$ is closely associated to $\mathrm{MI} / \mathrm{R}$ induced inflammation (39). Endothelial-derived NO can
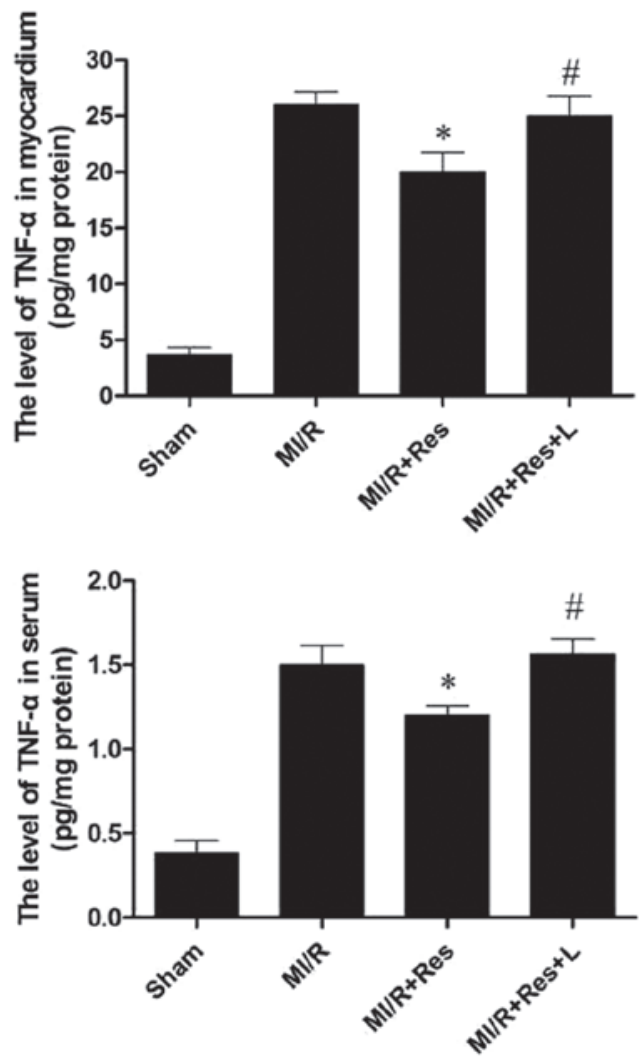

Figure 6. Comparison of expression levels of TNF- $\alpha$ in the myocardium and serum in each group. As compared with the MI/R group, resveratrol reduced TNF- $\alpha$ expression levels significantly. L-NAME abolished the effects of resveratrol. Error bars represent the mean \pm standard deviation. ${ }^{*} \mathrm{P}<0.05$ vs. Sham, ${ }^{\#} \mathrm{P}<0.05$ vs. MI/R. Sham, sham group; MI/R, MI/R group; $\mathrm{MI} / \mathrm{R}+\mathrm{Res}, \mathrm{MI} / \mathrm{R}+$ resveratrol group; $\mathrm{MI} / \mathrm{R}+\mathrm{Res}+\mathrm{L}-\mathrm{NAME}, \mathrm{MI} / \mathrm{R}$ + resveratrol + L-NAME group; MI, myocardial ischemia; R, reperfusion; Res, resveratrol.

inhibit cell adhesion factors, such as P-selectin and ICAM-1 levels, thereby inhibiting leukocyte adhesion and inward membrane migration (40). Endothelial-derived NO can inhibit the expression of TNF- $\alpha$ and other pro-inflammatory factors. In addition, endothelial-derived NO can increase the expression levels of IL-10 and other anti-inflammatory factors, and indirectly inhibit aggregation of inflammatory cells during local inflammation, thereby reducing the inflammatory response (41). In the present study, administration of L-NAME, a nitric oxide synthase inhibitor, abolished the protective effect of Res. This suggested that NO functions in the protective role of Res. Similarly, when methylene blue, a cGMP inhibitor, was added, the protective action of Res was blocked, indicating that cGMP pathway also functions in the protective role of Res.

Previous research has shown that SIRT1 confers protection in various models of cardiovascular oxidative stress (42-44). SIRT1 plays a critical role in endothelial homeostasis through regulation of the endothelial NOS (eNOS). Endothelial-derived NO regulates blood vessel relaxation and provides atheroprotective effects. Res, a polyphenolic activator of SIRT1, has been shown to increase the expression of eNOS (45) and the combination of Res with the HMG-CoA reductase inhibitors (statins) increased the activation of eNOS resulting in an increased functional recovery 
in a model of acute myocardial infarction (46). Additionally, chronic Res treatment improved endothelium-dependent relaxation in spontaneous hypertensive rats; however, it did not increase eNOS expression (47). The role of SIRT1 in the cardioprotective process of Res remains controversial following recent reports that have suggested that Res is not a direct activator of SIRT1 (48). The underlying mechanisms by which Res enhances SIRT1 activity remains poorly defined and requires further study (49).

In conclusion, the present study demonstrated that Res can attenuate inflammation induced by $\mathrm{MI} / \mathrm{R}$ injury through the TLR4/NF- $\kappa \mathrm{B}$ signaling pathway. The protective effects of Res are closely associated with the increase of NO production, the inhibition of neutrophil infiltration and TNF- $\alpha$ production.

\section{Acknowledgements}

This work was supported by funding from the National Natural Science Foundation of China (no. 81350026); the Scientific Research Fund of the Heilongjiang Provincial Education Department (no. 11551204); and the Natural Science Foundation of Heilongjiang Province, China (no. H201344).

\section{References}

1. Xiong J, Xue FS, Yuan YJ, et al: Cholinergic anti-inflammatory pathway: a possible approach to protect against myocardial ischemia reperfusion injury. Chin Med J (Engl) 123: 2720-2726, 2010.

2. Naidu BV, Farivar AS, Woolley SM, et al: Novel broad-spectrum chemokine inhibitor protects against lung ischemia-reperfusion injury. J Heart Lung Transplant 23: 128-134, 2004.

3. Batista ML Jr, Rosa JC, Lopes RD, et al: Exercise training changes IL-10/TNF-alpha ratio in the skeletal muscle of post-MI rats. Cytokine 49: 102-108, 2010.

4. Shimamoto A, Chong AJ, Yada M, et al: Inhibition of Toll-like receptor 4 with eritoran attenuates myocardial ischemia-reperfusion injury. Circulation 114: I270-I274, 2006.

5. Kawai T and Akira S: Signaling to NF-kappaB by Toll-like receptors. Trends Mol Med 13: 460-469, 2007.

6. Kaisho T and Akira S: Toll-like receptor function and signaling. J Allergy Clin Immunol 117: 979-987, 2006.

7. Arumugam TV, Okun E, Tang SC, et al: Toll-like receptors in ischemia-reperfusion injury. Shock 32: 4-16, 2009.

8. Kaczorowski DJ, Nakao A, McCurry KR and Billiar TR: Toll-like receptors and myocardial ischemia/reperfusion, inflammation, and injury. Curr Cardiol Rev 5: 196-202, 2009.

9. Smiley ST, King JA and Hancock WW: Fibrinogen stimulates macrophage chemokine secretion through Toll-like receptor 4 J Immunol 167: 2887-2894, 2001.

10. Yu M, Wang H, Ding A, et al: HMGB1 signals through Toll-like receptor (TLR) 4 and TLR2. Shock 26: 174-179, 2006.

11. Imai Y, Kuba K, Neely GG, et al: Identification of oxidative stress and Toll-like receptor 4 signaling as a key pathway of acute lung injury. Cell 133: 235-249, 2008.

12. Frantz S, Kobzik L, Kim YD, et al: Toll4 (TLR4) expression in cardiac myocytes in normal and failing myocardium. J Clin Invest 104: 271-280, 1999.

13. Renaud S and de Lorgeril M: Wine, alcohol, platelets, and the French paradox for coronary heart disease. Lancet 339 : $1523-1526,1992$.

14. Athar M, Back JH, Tang X, et al: Resveratrol: A review of preclinical studies for human cancer prevention. Toxicol Appl Pharmacol 224: 274-283, 2007.

15. Aluyen JK, Ton QN, Tran T, et al: Resveratrol: potential as anticancer agent. J Diet Suppl 9: 45-56, 2012.

16. Piotrowska H, Myszkowski K, Ziółkowska A, et al: Resveratrol analogue 3,4, 4', 5-tetramethoxystilbene inhibits growth, arrests cell cycle and induces apoptosis in ovarian SKOV-3 and A-2780 cancer cells. Toxicol Appl Pharmacol 263: 53-60, 2012.
17. Afaq F, Adhami VM and Ahmad N: Prevention of short-term ultraviolet $\mathrm{B}$ radiation-mediated damages by resveratrol in SKH-1 hairless mice. Toxicol Appl Pharmacol 186: 28-37, 2003.

18. Sehirli O, Sakarcan A, Velioğlu-Oğünç A, et al: Resveratrol improves ifosfamide-induced Fanconi syndrome in rats. Toxicol Appl Pharmacol 222: 33-41, 2007.

19. Xu Y, Nie L, Yin YG, et al: Resveratrol protects against hyperglycemia-induced oxidative damage to mitochondria by activating SIRT1 in rat mesangial cells. Toxicol Appl Pharmacol 259: 395-401, 2012.

20. Li F, Gong Q, Dong H and Shi J: Resveratrol, a neuroprotective supplement for Alzheimer's disease. Curr Pharm Des 18: 27-33, 2012.

21. López-Miranda V, Soto-Montenegro ML, Vera G, et al: Resveratrol: a neuroprotective polyphenol in the Mediterranean diet. Rev Neurol 54: 349-356, 2012 (In Spanish).

22. Borriello A1, Bencivenga D, Caldarelli I, et al: Resveratrol: from basic studies to bedside. Borriello A, Bencivenga D, Caldarelli I, Tramontano A, Borgia A, Zappia V, Della Ragione F. Cancer Treat Res 159: 167-184, 2014.

23. Black SC and Rodger IW: Methods for studying experimental myocardial ischemic and reperfusion injury. J Pharmacol Toxicol Methods 35: 179-190, 1996

24. Su H, Sun X, Ma H, et al: Acute hyperglycemia exacerbates myocardial ischemia/reperfusion injury and blunts cardioprotective effect of GIK. Am J Physiol-Endoc Metab 293: E629-E635, 2007.

25. Speyer CL and Ward PA: Role of endothelial chemokines and their receptors during inflammation. J Invest Surg 24: 18-27, 2011.

26. Khimenko PL, Bagby GJ, Fuseler J and Taylor AE: Tumor necrosis factor-alpha in ischemia and reperfusion injury in rat lungs. J Appl Physiol (1985) 85: 2005-2011, 1998.

27. Batista ML, Rosa JC, Lopes RD, et al: Exercise training changes IL-10/TNF-alpha ratio in the skeletal muscle of post-MI rats. Cytokine 49: 102-108, 2010.

28. Zhu J, Liu M, Kennedy RH and Liu SJ: TNF-alpha-induced impairment of mitochondrial integrity and apoptosis mediated by caspase- 8 in adult ventricular myocytes. Cytokine 34: 96-105, 2006.

29. Meldrum DR, Cleveland JC Jr, Cain BS, Meng X and Harken AH: Increased myocardial tumor necrosis factor-alpha in a crystalloid-perfused model of cardiac ischemia-reperfusion injury. Ann Thorac Surg 65: 439-443, 1998.

30. Gurevitch J, Frolkis I, Yuhas Y, et al: Antitumor necrosis factor-alpha improves myocardial recovery after ischemia and reperfusion. J Am Coll Cardiol 30: 1554-1561, 1997.

31. Lefer AM, Ma XL, Weyrich A and Lefer DJ: Endothelial dysfunction and neutrophil adherence as critical events in the development of reperfusion injury. Agents Actions Suppl 41: 127-135, 1993.

32. Ma XL, Lefer DJ, Lefer AM and Rothlein R: Coronary endothelial and cardiac protective effects of a monoclonal antibody to intercellular adhesion molecule-1 in myocardial ischemia and reperfusion. Circulation 86: 937-946, 1992.

33. Chandrasekar B,Smith JB and Freeman GL: Ischemia-reperfusion of rat myocardium activates nuclear factor-KappaB and induces neutrophil infiltration via lipopolysaccharide-induced CXC chemokine. Circulation 103: 2296-2302, 2001.

34. Otsui K, Inoue N, Kobayashi S, et al: Enhanced expression of TLR4 in smooth muscle cells in human atherosclerotic coronary arteries. Heart Vessels 22: 416-422, 2007.

35. Ha T, Li Y, Hua F, et al: Reduced cardiac hypertrophy in Toll-like receptor 4-deficient mice following pressure overload. Cardiovasc Res 68: 224-234, 2005.

36. Takahashi T: Toll-like receptors and myocardial contractile dysfunction. Cardiovasc Res 78: 3-4, 2008.

37. Hoshino K, Takeuchi O, Kawai T, et al: Cutting edge: Toll-like receptor 4 (TLR4)-deficient mice are hyporesponsive to lipopolysaccharide: evidence for TLR4 as the Lps gene product. J Immunol 162: 3749-3752, 1999.

38. Liu YY, Cai WF, Yang HZ, et al: Bacillus Calmette-Guérin and TLR4 agonist prevent cardiovascular hypertrophy and fibrosis by regulating immune microenvironment. J Immunol 180: 7349-7357, 2008

39. Liu P, Hock CE, Nagele R and Wong PY: Formation of nitric oxide, superoxide, and peroxynitrite in myocardial ischemia-reperfusion injury in rats. Am J Physiol 272: H2327-H2336, 1997. 
40. Li J, Wu F, Zhang H, et al: Insulin inhibits leukocyte-endothelium adherence via an Akt-NO-dependent mechanism in myocardial ischemia/reperfusion. J Mol Cell Cardiol 47: 512-519, 2009.

41. Li J, Zhang H, Wu F, et al: Insulin inhibits tumor necrosis factor-alpha induction in myocardial ischemia/reperfusion: role of Akt and endothelial nitric oxide synthase phosphorylation. Crit Care Med 36: 1551-1558, 2008.

42. Alcendor RR, Gao S, Zhai P, et al: Sirt1 regulates aging and resistance to oxidative stress in the heart. Circ Res 100: 1512-1521, 2007.

43. Danz ED, Skramsted J, Henry N, Bennett JA and Keller RS: Resveratrol prevents doxorubicin cardiotoxicity through mitochondrial stabilization and the Sirt1 pathway. Free Radic Biol Med 46: 1589-1597, 2009.

44. Pillai JB, Isbatan A, Imai S and Gupta MP: Poly (ADP-ribose) polymerase-1-dependent cardiac myocyte cell death during heart failure is mediated by NAD+ depletion and reduced Sir2alpha deacetylase activity. J Biol Chem 280: 43121-43130, 2005.

45. Wallerath T, Li H, Gödtel-Ambrust U, Schwarz PM and Forstermann UA: Blend of polyphenolic compounds explains the stimulatory effect of red wine on human endothelial NO synthase. Nitric Oxide 12: 97-104, 2005.
46. Penumathsa SV, Thirunavukkarasu M, Koneru S, et al: Statin and resveratrol in combination induces cardioprotection against myocardial infarction in hypercholesterolemic rat. J Mol Cell Cardiol 42: 508-516, 2007.

47. Rush JW, Quadrilatero J, Levy AS and Ford RJ: Chronic resveratrol enhances endothelium-dependent relaxation but does not alter eNOS levels in aorta of spontaneously hypertensive rats. Exp Biol Med (Maywood) 232: 814-822, 2007.

48. Beher D, Wu J, Cumine S, et al: Resveratrol is not a direct activator of SIRT1 enzyme activity. Chem Biol Drug Des 74: 619-624, 2009

49. Chakrabarty SP, Balaram H and Chandrasekaran S: Sirtuins: multifaceted drug targets. Curr Mol Med 11: 709-718, 2011. Danz ED, Skramsted J, Henry N, Bennett JA and Keller RS: Resveratrol prevents doxorubicin cardiotoxicity through mitochondrial stabilization and the Sirtl pathway. Free Radic Biol Med 46: 1589-1597, 2009. 